\title{
MODIFIED CAUCHY KERNELS AND FUNCTIONAL CALCULUS FOR OPERATORS ON BANACH SPACE
}

\author{
EDWIN FRANKS
}

(Received 29 January 1996; revised 23 August 1996)

Communicated by E. N. Dancer

\begin{abstract}
In Banach space operators with a bounded $H^{\infty}$ functional calculus, Cowling et al. provide some necessary and sufficient conditions for a type- $\omega$ operator to have a bounded $H^{\infty}$ functional calculus. We provide an alternate development of some of their ideas using a modified Cauchy kernel which is $L^{1}$ with respect to the measure $|d z| /|z|$. The method is direct and has the advantage that no transforms of the functions are necessary.
\end{abstract}

1991 Mathematics subject classification (Amer. Math. Soc.): primary 47A60.

Keywords and phrases: functional calculus, matricial functional calculus.

\section{Introduction}

In [1] Cowling et al. show that type- $\omega$ operators (defined below) on Banach spaces which satisfy 'weak quadratic estimates' possess a bounded functional calculus for holomorphic functions. In Section 2 of this paper we obtain similar results using a modified Cauchy kernel applied to the Riesz-Dunford formula for functional calculi. In Section 3 we derive some 'strong quadratic estimates', a special case of those in [1], which are sufficient for a type- $\omega$ operator on $L^{p}(\Omega)$ to have a bounded $H^{\infty}$ functional calculus. The derivation is almost immediate from the results in Section 2 and allows us in Section 4 to show that type- $\omega$ operators on Hilbert spaces which possess bounded functional calculi for functions also admit uniformly bounded functional calculi for matrices. This implies using the results of Paulsen [9], that such operators are similar to operators with functional calculus constant 1 . This result has been obtained independently by Christian Le Merdy [8].

We begin with some notation, definitions, and assumptions. Throughout, $X$ and

(C) 1997 Australian Mathematical Society 0263-6115/97\$A2.00+0.00 
$\mathscr{H}$ denote complex Banach spaces and complex Hilbert spaces respectively. All operators $T$ acting on $X$ or $\mathscr{H}$ will be assumed to be closed, one-one and have dense domains and dense ranges. For $0 \leq \mu<\pi$, let $S_{\mu}$ denote the open sector of angle $\mu$, that is, $S_{\mu}=\{z \in \mathbb{C}:|\arg z|<\mu\}$. Let $H^{\infty}\left(S_{\mu}\right)$ denote the space of functions which are bounded and holomorphic on $S_{\mu}$. For $h \in H^{\infty}\left(S_{\mu}\right)$ set $\|h\|_{\infty}=\sup _{z \in S_{\mu}}|h(z)|$.

We are interested in operators which satisfy the following condition on their resolvents.

DEFINITION 1.1. An operator $T$ in $X$ is said to be type- $\omega$, where $0 \leq \omega<\pi$, if $T$ is closed, $\sigma(T) \subseteq S_{\omega} \cup \partial S_{\omega}$, and for each $\mu$ in $(\omega, \pi)$ and $z$ in $\mathbb{C} \backslash S_{\mu}$,

$$
\left\|(T-z)^{-1}\right\| \leq c|z|^{-1} .
$$

One-one type- $\omega$ operators always possess an $H^{\infty}$ functional calculus. That is, for $\mu>\omega$ there exists a unique algebra homomorphism from $H^{\infty}\left(S_{\mu}\right)$ into the space of closed operators on $X$ which takes $(\lambda-z)^{-1}$ to $(\lambda-T)^{-1}$. However it may happen that for some $h \in H^{\infty}\left(S_{\mu}\right)$ with $\|h\|_{\infty}=1$ we have $\|h(T)\|=\infty$; see [6, 7]. (An operator with this property may also be obtained by taking the Cayley transform of Foguel's 1964 counterexample $[2,5]$.) To show that the conditions we derive in Sections 2 and 3 guarantee a bounded functional calculus, we shall need McIntosh's result for approximating operators, namely the Convergence Lemma.

LEMMA 1.2 (Convergence Lemma). Suppose $T$ is an operator of type- $\omega$ which is one-to-one with dense domain and dense range in $X$, and that $\mu>\omega$. Let $\left\{f_{\alpha}\right\}$ be a uniformly bounded net of functions in $H^{\infty}\left(S_{\mu}\right)$ which converges to a function $f$ in $H^{\infty}\left(S_{\mu}\right)$ uniformly on compact subsets of $S_{\mu}$. Suppose further that the operators $f_{\alpha}(T)$ are uniformly bounded on $X$. Then $f_{\alpha}(T) u$ converges to $f(T) u$ for all $u$ in $X$, and consequently $f(T)$ is a bounded linear operator on $X$, and $\|f(T)\| \leq \sup _{\alpha}\left\|f_{\alpha}(T)\right\|$.

We use here the 'variable constant convention', according to which $c, c_{1}, \ldots$, denote constants (in $\mathbb{R}^{+}$) which may vary from one occurrence to the next. In a given formula, the constant does not depend on variables expressly quantified after the formula, but it may depend on variables quantified (implicitly or explicitly) before. Thus, in Definition 1.1, $c$ may depend on $X, T, \omega$, and $\mu$, but not on $z$.

\section{2. $H^{\infty}$ functional calculus in Banach spaces}

Let $T$ be a one-one type- $\omega$ operator acting in a complex Banach space $X$ having dense domain and dense range. Let $\langle\cdot, \cdot\rangle$ denote the bilinear pairing between $X$ and $X^{*}$. We wish to describe some necessary and sufficient conditions for $T$ to 
have a bounded $H^{\infty}$ functional calculus. Our method uses a modification of the Cauchy kernel and an associated modification in the definition of the Riesz-Dunford functional calculus. Initially to simplify matters we restrict our attention to functions in $H^{\infty}\left(S_{\mu}\right) \cap L^{1}\left(\partial S_{\mu},|d z| /|z|\right)$. For $\mu>\omega, h \in H^{\infty}\left(S_{\mu}\right) \cap L^{1}\left(\partial S_{\mu},|d z| /|z|\right)$ and $\zeta$ in the interior of $S_{\mu}$,

$$
h(\zeta)=\frac{1}{2 \pi i} \int_{\partial S_{\mu}} \frac{h(z)}{(z-\zeta)} d z
$$

and

$$
h(T)=\frac{1}{2 \pi i} \int_{\partial S_{\mu}} \frac{h(z)}{(z-T)} d z
$$

(Since $T$ is type- $\omega$, the second integral converges absolutely in operator norm.)

However, many other kernels besides $(z-\zeta)^{-1}$ will reproduce the values of holomorphic functions and provide formulas for holomorphic functions of type- $\omega$ operators. For example, Cauchy's theorem shows that,

$$
h(\zeta)=\frac{1}{2 \pi i} \int_{\partial S_{\mu}} \frac{h(z) z^{1 / 2} \zeta^{1 / 2}}{(z-\zeta)} \frac{d z}{z}=\frac{1}{2 \pi i} \int_{\partial S_{\mu}} \frac{2 h(z) z \zeta}{\left(z^{2}-\zeta^{2}\right)} \frac{d z}{z},
$$

and

$$
h(T)=\frac{1}{2 \pi i} \int_{\partial S_{\mu}} \frac{h(z) z^{1 / 2} T^{1 / 2}}{(z-T)} \frac{d z}{z}=\frac{1}{2 \pi i} \int_{\partial S_{\mu}} \frac{2 h(z) z T}{\left(z^{2}-T^{2}\right)} \frac{d z}{z}
$$

Note that for the second equalities to hold in the above, $\mu<\pi / 2$. Also, using (1), one sees that $\left\|z^{1 / 2} T^{1 / 2}(z-T)^{-1}\right\|$ and $\left\|z T\left(z^{2}-T^{2}\right)^{-1}\right\|$ are uniformly bounded in $z$, so that the integrals in (3) converge absolutely in the operator norm.

In these formulas the measure $d z$ has been replaced by $d z / z$ and the kernels have changed. The advantage is, that while $(z-\zeta)^{-1}$ is not integrable with respect to $|d z|$ on $\partial S_{\mu}$, both $z^{1 / 2} \zeta^{1 / 2}(z-\zeta)^{-1}$, and $2 z \zeta\left(z^{2}-\zeta^{2}\right)^{-1}$ are integrable with respect to $|d z| /|z|$. This allows one to unambiguously extend the formulas in (2) to all of $H^{\infty}\left(S_{\mu}\right)$. Furthermore, the $L^{1}$ norms of these kernels depend only on the argument of $\zeta$, a fact critical when $\zeta$ is replaced by an operator $T$ in order to make estimates about the norm of $h(T)$.

Fix $v>\omega, h \in H^{\infty}\left(S_{v}\right) \cap L^{1}\left(\partial S_{v},|d z| /|z|\right), u \in X$, and $v \in X^{*}$ with $\|u\|=$ $\|v\|=1$. Using (3) one has 


$$
\begin{aligned}
|\langle h(T) u, v\rangle| & =\left|\frac{1}{2 \pi i} \int_{\partial S_{v}}\left\langle\frac{h(z) z^{1 / 2} T^{1 / 2}}{(z-T)} u, v\right\rangle \frac{d z}{z}\right| \\
& \leq \frac{1}{2 \pi} \int_{\partial S_{v}}\left|\left\langle\frac{h(z) z^{1 / 2} T^{1 / 2}}{(z-T)} u, v\right\rangle\right| \frac{|d z|}{|z|} \\
& \leq \frac{1}{2 \pi}\|h\|_{\infty} \int_{\partial S_{v}}\left|\left\langle\frac{z^{1 / 2} T^{1 / 2}}{(z-T)} u, v\right\rangle\right| \frac{|d z|}{|z|} .
\end{aligned}
$$

Hence if for all $u \in X$, and $v \in X^{*}$,

$$
\int_{\partial S_{v}}\left|\left\langle\frac{z^{1 / 2} T^{1 / 2}}{(z-T)} u, v\right\rangle\right| \frac{|d z|}{|z|} \leq c\|u\|\|v\|,
$$

then $\|h(T)\| \leq c\|h\|_{\infty}$ for all $h \in H^{\infty}\left(S_{v}\right) \cap L^{1}\left(\partial S_{v},|d z| /|z|\right)$. This implies via the Convergence Lemma that $T$ has a bounded $H^{\infty}\left(S_{v}\right)$ functional calculus. In fact we have the following theorem which is a version of [1, Theorems 4.2 and 4.4].

THEOREM 2.1. Let $T$ be a one-one type- $\omega$ operator acting in a complex Banach space $X$ having dense domain and dense range. If (5) holds for all $u \in X$ and $v \in X^{*}$ then $T$ has a bounded $H^{\infty}\left(S_{v}\right)$ functional calculus. Conversely if $T$ has a bounded $H^{\infty}\left(S_{\mu}\right)$ functional calculus, then (5) holds for all $u \in X$, and $v \in X^{*}$.

PROOF. We have proven the first part. To see the second part, fix $v>\mu>\omega$, $u \in X$, and $v \in X^{*}$. For $z \in \partial S_{v}$, let $a(z, u, v)$ be the unimodular function determined by the relation

$$
\frac{1}{2 \pi i} \int_{\partial S_{v}} a(z, u, v)\left\langle\frac{z^{1 / 2} T^{1 / 2}}{(z-T)} u, v\right\rangle \frac{d z}{z}=\frac{1}{2 \pi} \int_{\partial S_{v}}\left|\left\langle\frac{z^{1 / 2} T^{1 / 2}}{(z-T)} u, v\right\rangle\right| \frac{|d z|}{|z|} .
$$

For $\zeta \in S_{\mu}$, define a holomorphic function $F_{u, v^{v}}(\zeta)$ by the formula,

$$
F_{u, v}(\zeta)=\int_{\partial S_{v}} a(z, u, v) \frac{z^{1 / 2} \zeta^{1 / 2}}{(z-\zeta)} \frac{d z}{z}
$$

One easily sees that

$$
\sup _{\zeta \in S_{\mu}}\left|F_{u, v}(\zeta)\right| \leq c \frac{1}{(\nu-\mu)}
$$

Now using (4) and (6) one has that

$$
\int_{\partial S_{v}}\left|\left\langle\frac{z^{1 / 2} T^{1 / 2}}{(z-T)} u, v\right\rangle\right| \frac{|d z|}{|z|}=\left\langle F_{u, v, h}(T) u, v\right\rangle,
$$

and thus by (7), (5) holds if $T$ has a bounded $H^{\infty}\left(S_{\mu}\right)$ functional calculus. 
To see that Theorem 2.1 is similar to [1, Theorems 4.2 and 4.4] note that with a suitable change of variables the integral in (5) can be written as two integrals over $\mathbb{R}^{+}$ with respect to $d t / t$, corresponding to the upper and lower rays of $\partial S_{\mu}$. Then, upon replacing $t$ by $1 / t$, the kernel $z^{1 / 2} T^{1 / 2}(z-T)^{-1}$ becomes,

$$
\psi_{+}(t T)=e^{i v / 2} t^{1 / 2} T^{1 / 2} /\left(e^{i v}-t T\right)
$$

for the upper ray, and

$$
\psi_{-}(t T)=e^{-i v / 2} t^{1 / 2} T^{1 / 2} /\left(e^{-i v}-t T\right)
$$

for the lower ray. Note that for all $z \in S_{\mu}$ one has

$$
\left|\psi_{+.-}(z)\right| \leq c|z|^{1 / 2} /(1+|z|)
$$

thus $\psi_{+,-}(z) \in \Psi\left(S_{\mu}\right)$ as defined in [1]. Rewriting (5) we obtain,

$$
\int_{0}^{\infty}\left(\left|\left\langle\psi_{+}(t T) u, v\right\rangle\right|+\left|\left\langle\psi_{-}(t T) u, v\right\rangle\right|\right) \frac{d t}{t} \leq c\|u\|\|v\| .
$$

\section{3. $H^{\infty}$ functional calculus for operators on $L^{p}$.}

We now wish to apply the modified Cauchy kernels to $L^{p}$ spaces. Accordingly, let $\Omega$ be a domain in $\mathbb{R}^{n}$ and let $T$ be a one-one type- $\omega$ operator acting on $L^{p}(\Omega)$ having dense domain and dense range. Let $q$ be the conjugate exponent to $p$ and let $T^{*}$ be the adjoint of $T$ with respect to the bilinear pairing $\langle\cdot, \cdot\rangle$ between $L^{p}$ and $L^{q}$. Let $\psi_{+.-}(t T)$ be defined by formulas (8) and (9) above. Fix $v>\mu>\omega$, $f \in H^{\infty}\left(S_{\nu}\right) \cap L^{1}\left(\partial S_{\mu},|d z| /|z|\right), u \in L^{p}, v \in L^{q}$, and $\zeta \in S_{\mu}$. Using Section 2 and the above definitions one has,

$$
\begin{aligned}
|\langle f(T) u, v\rangle| & \leq \frac{1}{2 \pi} \int_{\partial S_{\mu}}\left|\left\langle\frac{f(z) z^{1 / 2} T^{1 / 2}}{(z-T)} u, v\right\rangle\right| \frac{|d z|}{|z|} \\
& \leq c\|f\|_{\infty} \int_{0}^{\infty}\left(\left|\left\langle\psi_{+}(t T) u, v\right\rangle\right|+\left|\left\langle\psi_{-}(t T) u, v\right\rangle\right|\right) d t / t \\
& =c\|f\|_{\infty} \int_{0}^{\infty}\left(\left|\left\langle\psi_{+}^{1 / 2}(t T) u, \psi_{+}^{1 / 2}\left(t T^{*}\right) v\right\rangle\right|+\left|\left\langle\psi_{-}^{1 / 2}(t T) u, \psi_{-}^{1 / 2}\left(t T^{*}\right) v\right\rangle\right|\right) d t / t
\end{aligned}
$$

Considering just the first term of this last integral and using the fact that we are working in function space gives, 


$$
\begin{aligned}
\int_{0}^{\infty} & \left|\left\langle\psi_{+}^{1 / 2}(t T) u, \psi_{+}^{1 / 2}\left(t T^{*}\right) v\right\rangle\right| d t / t \\
& \leq \int_{\Omega} \int_{0}^{\infty}\left|\psi_{+}^{1 / 2}(t T) u(x) \psi_{+}^{1 / 2}\left(t T^{*}\right) v(x)\right| \frac{d t}{t} d x \\
& \leq \int_{\Omega}\left\{\int_{0}^{\infty}\left|\psi_{+}^{1 / 2}(t T) u(x)\right|^{2} \frac{d t}{t}\right\}^{1 / 2}\left\{\iint_{0}^{\infty}\left|\psi_{+}^{1 / 2}\left(t T^{*}\right) v(x)\right| \frac{d t}{t}\right\}^{1 / 2} d x \\
& \leq\left\|\left\{\int_{0}^{\infty}\left|\psi_{+}^{1 / 2}(t T) u(\cdot)\right|^{2} \frac{d t}{t}\right\}^{1 / 2}\right\|\left\|\left\{\int_{0}^{\infty}\left|\psi_{+}^{1 / 2}\left(t T^{*}\right) v(\cdot)\right| \frac{d t}{t}\right\}^{1 / 2}\right\|_{q}
\end{aligned}
$$

This last quadratic expression, which we have arrived at without intricate transforms and estimates, is a special case of the quadratic expressions [1]. Using the above and the Convergence Lemma one can prove the following version of [1, Corallary 6.8]:

THEOREM 3.1. Let $T$ be a one-one type- $\omega$ operator acting on $L^{p}(\Omega)$ having dense domain and dense range. If for all $u \in L^{p}$ and $v \in L^{q}$,

(11)

$$
\left\|\left\{\int_{0}^{\infty}\left|\psi_{+}^{1 / 2}(t T) u(\cdot)\right|^{2} \frac{d t}{t}\right\}^{1 / 2}\right\|_{p}+\left\|\left\{\int_{0}^{\infty}\left|\psi_{-}^{1 / 2}(t T) u(\cdot)\right|^{2} \frac{d t}{t}\right\}^{1 / 2}\right\|_{p} \leq c\|u\|_{p},
$$

and

$$
\left\|\left\{\int_{0}^{\infty}\left|\psi_{+}^{1 / 2}\left(t T^{*}\right) v(\cdot)\right| \frac{d t}{t}\right\}^{1 / 2}\right\|_{q}+\left\|\left\{\int_{0}^{\infty}\left|\psi_{-}^{1 / 2}\left(t T^{*}\right) v(\cdot)\right| \frac{d t}{t}\right\}^{1 / 2}\right\|_{q} \leq c\|v\|_{q}
$$

then $T$ has a bounded $H^{\infty}\left(S_{v}\right)$ functional calculus.

The converse of this theorem also holds but the methods employed here will not yield a proof. For completeness, and use in Section 4, we state the converse proved in [1].

THEOREM 3.2. Let $T$ be a one-one type- $\omega$ operator acting on $L^{p}(\Omega)$ having dense domain and dense range. Suppose $T$ has a bounded $H^{\infty}\left(S_{\mu}\right)$ functional calculus. Then for all $u \in L^{p}$ and $v \in L^{q}$, (11) and (12) hold.

(Note that the angles have changed so that this is not a precise converse.) 


\section{4. $H^{\infty}$ matricial functional calculus}

Let $\mathscr{H}$ be a complex Hilbert space. In this section we show how by doing little more than adding indices to the formulas in the previously sections one can extend the bounded $H^{\infty}\left(S_{v}\right)$ functional calculus for an operator $T$ acting $\mathscr{H}$ to a bounded $H^{\infty}\left(S_{\nu} ; \mathbb{C}^{n \times n}\right)$ functional calculus. Let $(\mathscr{H})^{(n)}$ denote the space of $n$-tuples of vectors in $\mathscr{H}$. For $u \in(\mathscr{H})^{(n)}$ set $\|u\|_{2,2}=c\left(\sum_{m=1}^{n}\left\|u_{m}\right\|_{2}^{2}\right)^{1 / 2}$.

With this definition we have the following theorem.

THEOREM 4.1. Let $T$ be a one-one type- $\omega$ operator acting on a complex Hilbert space $\mathscr{H}$ having dense domain and dense range. Suppose $T$ has a bounded $H^{\infty}\left(S_{\mu}\right)$ functional calculus. Then for $n=1,2, \ldots,\left[f_{i, j}(z)\right] \in H^{\infty}\left(S_{v} ; \mathbb{C}^{n \times n}\right)$, and $u \in$ $\left(L^{2}\right)^{(n)}$,

$$
\left|\left\langle\left[f_{i, j}(T)\right] u, u\right\rangle\right| \leq c\left\|\left[f_{i, j}\right]\right\|_{\infty}\|u\|_{2,2}^{2},
$$

where $c$ does not depend on $n$.

PROOF. To show $T$ has a bounded $H^{\infty}\left(S_{v} ; \mathbb{C}^{n \times n}\right)$ functional calculus it suffices to consider $T$ restricted to a closed separable invariant subspace generated by a set of $n$ vector $\Gamma=\left\{\gamma_{1}, \gamma_{2}, \ldots, \gamma_{n}\right\}$ and the functional calculus of $T$ : namely, the closure of a subspace of the form $\mathscr{H}_{\Gamma}=\left\{\sum_{i=1}^{n} h_{i}(T) \gamma_{i}: h_{i} \in H^{\infty}\left(S_{\mu}\right), 1 \leq i \leq n\right\}$. Thus, since any separable Hilbert space is unitarily equivalent to $L^{2}(\Omega)$ for some $\Omega \subset \mathbb{R}^{n}$, we may assume without loss of generality that $\mathscr{H}=L^{2}(\Omega)$.

Fix $\left[f_{i, j}(z)\right] \in H^{\infty}\left(S_{v} ; \mathbb{C}^{n \times n}\right)$, and $u \in\left(L^{2}\right)^{(n)}$. We assume also that

$$
\left\|\left[f_{i, j}\right]\right\|_{\infty} \leq 1
$$

Estimating in an analogous fashion to the previous section one has

$$
\begin{aligned}
& \left|\left\langle\left[f_{i, j}(T)\right] u, u\right\rangle\right| \leq \frac{1}{2 \pi} \int_{\partial S_{\mu}} \mid\left\langle\frac{\left.{\frac{z}{1 / 2} T^{1 / 2}}_{(z-T)}\left[f_{i, j}(z)\right] u, u\right\rangle \mid \frac{|d z|}{|z|}}{\leq c \int_{0}^{\infty}\left(\left|\left\langle\psi_{+}(t T)\left[f_{i, j}\left(e^{i v} t^{-1}\right)\right] u, u\right\rangle\right|+\left|\left\langle\psi_{-}(t T)\left[f_{i, j}\left(e^{-i v} t^{-1}\right)\right] u, u\right\rangle\right|\right) \frac{d t}{t}}\right. \\
& =c \int_{0}^{\infty}\left(\left|\left\langle\psi_{+}^{1 / 2}(t T)\left[f_{i, j}\left(e^{i v} t^{-1}\right)\right] u, \psi_{+}^{1 / 2}\left(t T^{*}\right) u\right\rangle\right|\right. \\
& \left.\quad+\left|\left\langle\psi_{-}^{1 / 2}(t T)\left[f_{i, j}\left(e^{-i v} t^{-1}\right)\right] u, \psi_{-}^{1 / 2}\left(t T^{*}\right) u\right\rangle\right|\right) \frac{d t}{t} .
\end{aligned}
$$

Now, using (13) and Hölder's inequality, one has that the first term of (14) is bounded by 


$$
\begin{aligned}
& \int_{\Omega} \int_{0}^{\infty}\left(\sum_{m=1}^{n}\left|\psi_{+}^{1 / 2}(t T) u_{m}(x)\right|^{2}\right)^{1 / 2}\left(\sum_{m=1}^{n}\left|\psi_{+}^{1 / 2}\left(t T^{*}\right) u_{m}(x)\right|^{2}\right)^{1 / 2} \frac{d t}{t} d x \\
& \leq c \int_{\Omega}\left\{\sum_{m=1}^{n} \int_{0}^{\infty}\left|\psi_{+}^{1 / 2}(t T) u_{m}(x)\right|^{2} \frac{d t}{t}\right\}^{1 / 2}\left\{\sum_{m=1}^{n} \int_{0}^{\infty}\left|\psi_{+}^{1 / 2}\left(t T^{*}\right) u_{m}(x)\right|^{2} \frac{d t}{t}\right\}^{1 / 2} d x \\
& \leq c\left\|\left\{\sum_{m=1}^{n} \int_{0}^{\infty}\left|\psi_{+}^{1 / 2}(t T) u_{m}(x)\right|^{2} \frac{d t}{t}\right\}^{1 / 2}\right\|_{2}\left\|\left\{\sum_{m=1}^{n} \int_{0}^{\infty}\left|\psi_{+}^{1 / 2}\left(t T^{*}\right) u_{m}(x)\right|^{2} \frac{d t}{t}\right\}^{1 / 2}\right\|_{2}
\end{aligned}
$$

Using Theorem 3.2 and the Convergence Lemma one has that the above and the analogous expression for $\psi_{-}^{1 / 2}$ are bounded by $\mathrm{c}\|u\|_{2,2}^{2}$, proving the theorem.

In [9] Paulsen showed that if $T$ has a uniformly bounded $H^{\infty}\left(S_{v} ; \mathbb{C}^{n \times n}\right)$ functional calculus then $T$ is similar to an operator $B$ with functional calculus constant 1 . That is, $T=L B L^{-1}$, where $L$ and $L^{-1}$ are bounded and $B$ satisfies $\|f(B)\| \leq\|f\|_{\infty}$. Thus Theorem 4.1 shows that if $T$ has a bounded $H^{\infty}\left(S_{\mu}\right)$ functional calculus, then $T$ is similar to an operator with functional calculus constant 1 . Note that, as mentioned in the introduction, this has been obtained independently by Le Merdy [8].

\section{Comments}

Theorems 2.1, 3.1 and 3.2 can be generalized in the manner alluded to in Section 2. The kernel $z^{1 / 2} \zeta^{1 / 2}(z-\zeta)^{-1}$ is not the only modification of the Cauchy kernel which reproduces the values of holomorphic functions and is integrable with respect to $|d z| /|z|$ on $\partial S_{v}$. Any other kernel with those properties would yield similar theorems $\left(2 z \zeta\left(z^{2}-\zeta^{2}\right)^{-1}\right.$ for example). The modification of the Cauchy kernel to get better integrability properties extends to the Clifford setting as well [4]. The author, in joint work with McIntosh, has developed an alternate and more direct approach to Theorem 3.2 , by discretizing the square function estimates on the sector [3].

\section{References}

[1] M. Cowling, I. Doust, A. McIntosh, and A. Yagi, 'Banach space operators with a bounded $H^{x}$ functional calculus', J. Austral. Math. Soc. (Series A) 60 (1996), 51-89.

[2] S. R. Foguel, 'A counterexample to a problem of Sz.-Nagy', Proc. Amer. Math. Soc. 15 (1964), 788-790.

[3] E. Franks and A. McIntosh, 'Discrete quadratic estimates and holomorphic functional calculi of operators in Banach spaces', preprint (1995). 
[4] E. Franks and J. Ryan, 'Bounded monogenic functions on unbounded domains', preprint (1995).

[5] P. R. Halmos, 'On Foguel's answer to Nagy's problem', Proc. Amer. Math. Soc. 15 (1964), 791-793.

[6] A. McIntosh, 'Operators which have an $H^{\infty}$ functional calculus', in: Miniconference on Operator Theory and Partial Differential Equations, Proc. Centre Math. Analysis, A.N.U., Canberra 14 (1986), 210-231.

[7] A. McIntosh and A. Yagi, 'Operators of type- $\omega$ without a bounded $H^{\infty}$ functional calculus', in: Miniconference on Operators in Analysis, Proc. Centre. Math. Analysis, A.N.U., Canberra 24 (1989), 159-172.

[8] C. Le Merdy, 'The similarity problem for bounded analytic semigroups on Hilbert space', preprint (1995).

[9] V. I. Paulsen, 'Every completely polynomially bounded operator is similar to a contraction', $J$. Funct. Anal. 55 (1984), 1-17.

Department of Mathematics

Macquarie University

NSW 2109

Australia

e-mail: edwin@mpce.mq.edu.au 